\title{
DECOMPOSIÇÃO E LIBERAÇÃO DE NUTRIENTES DE RESÍDUOS CULTURAIS DE PLANTAS DE COBERTURA EM ARGISSOLO VERMELHO-AMARELO NA REGIÃO NOROESTE FLUMINENSE (RJ) ${ }^{(1)}$
}

\author{
Antonio Carlos da Gama-Rodrigues ${ }^{(2)}$, Emanuela Forestieri da \\ Gama-Rodrigues $^{(3)}$ \& Elio Cruz de Brito ${ }^{(4)}$
}

\begin{abstract}
RESUMO
A decomposição pode assumir importante papel no manejo da fertilidade do solo, possibilitando a elaboração de técnicas de cultivo que melhorem a utilização de nutrientes contidos nos resíduos vegetais. $O$ objetivo deste trabalho foi estimar as taxas de decomposição e liberação de $\mathrm{C}, \mathrm{N}, \mathrm{P}, \mathrm{K}$, Ca e Mg de resíduos culturais provenientes de plantas de coberturas na cultura do maracujá. As espécies avaliadas foram feijão-de-porco (Canavalia ensiformis), amendoim forrageiro acesso CIAT 1734 (Arachis pintoi), siratro (Macroptilium atropurpureum), cudzu tropical (Pueraria phaseoloides) e Brachiaria brizantha. A decomposição dos resíduos culturais, colocados em sacos de malha de $2 \mathrm{~mm}$, foi avaliada durante 140 dias. $O$ modelo que proporcionou melhor ajuste foi o exponencial de primeira ordem. $O$ feijão-de-porco e o amendoim forrageiro apresentaram as maiores taxas de decomposição de matéria seca, diferindo significativamente das demais coberturas vegetais. As taxas de liberação de C, N, P, Ca e Mg foram maiores no feijão-de-porco. $\mathrm{O}$ amendoim forrageiro apresentou a maior taxa de liberação de K. Para todas as coberturas vegetais, os maiores valores médios de taxa de liberação foram de $\mathrm{K}$ e polifenóis. As taxas de liberação de C, N, P, Ca e Mg estão associadas positivamente à taxa de decomposição da matéria seca. As taxas de decomposição de matéria seca e de liberação de $C$, de nutrientes e de polifenóis variaram em função da qualidade nutricional e orgânica do substrato referente ao início do estudo. As distintas taxas de decomposição e liberação de nutrientes das espécies
\end{abstract}

\footnotetext{
(1) Recebido para publicação em maio de 2006 e aprovado em julho de 2007.

${ }^{(2)}$ Professor Associado do Laboratório de Solos, CCTA, Universidade Estadual do Norte Fluminense - UENF. CEP 28013-602 Campos dos Goytacazes (RJ). Bolsista FAPERJ. E-mail: tonygama@uenf.br

${ }^{(3)}$ Professora Associada do Laboratório de Solos, CCTA/UENF. E-mail: emanuela@uenf.br

${ }^{(4)}$ Mestre em Produção Vegetal, CCTA/UENF. E-mail: eliocruzbrito@hotmail.com
} 
estimadas mostraram o potencial de uso de resíduos vegetais como fonte de nutrientes na cultura do maracujá.

Termos de indexação: adubação verde, leguminosas, macronutrientes, qualidade química de resíduos vegetais.

\title{
SUMMARY: DECOMPOSITION AND NUTRIENT RELEASE FROM COVER CROP RESIDUES IN PASSION-FRUIT PLANTATION
}

\begin{abstract}
Decomposition can assume an important role in soil fertility management, underlying techniques that optimize the use of nutrients of plant residues. The objective of this study was to estimate the decomposition rate and nutrient release from cover crop residues in a passion-fruit plantation. The evaluated species were Canavalia ensiformis, Arachis pintoi, Macroptilium atropurpureum, Pueraria phaseoloides and Brachiaria brizantha. Litter decomposition was measured using nylon, $2.0 \mathrm{~mm}$ mesh, litter bags during 140 days. The single exponential model adjusted best for all cover crop species. Litter decay rates of Canavalia ensiformis and Arachis pintoi were significantly higher than in those of other species. The $C, N, P, C a$ and $M g$ release rates from Canavalia ensiformis residue were the highest. However, $K$ release rate was the highest for Arachis pintoi. For all residues the $K$ and polyphenol release rates were higher than those of the other elements. $C, N, P, C a$ and $M g$ release rates were positively correlated with litter decay rate. The litter decay rate, $C$, nutrients, and polyphenol release rates were regulated by litter quality. The different litter decay rates and estimated nutrient release rates indicate the potential of the use of crop residues as nutrient source of passion-fruit.
\end{abstract}

Index terms: green manure, legumes, carbon, litter quality.

\section{INTRODUÇÃO}

Na região noroeste fluminense, o longo período de atividades agropecuárias, especialmente da cana-deaçúcar e do café, com uso regular de fogo e mecanização intensiva resultou em elevado grau de degradação dos solos e, por conseguinte, na decadência socioeconômica da região, na qual as atividades de uso atual das terras são pastagens degradadas (Gama-Rodrigues \& May, 2001).

Em razão disso, a adoção de um sistema agroecológico torna-se uma necessidade de uso alternativo dessas terras degradadas, por maximizar a ação dos componentes bióticos no sistema de produção, mediante a reciclagem de nutrientes (Siqueira et al., 1999). Nesse sentido, o uso de plantas de cobertura do solo tem sido uma estratégia capaz de aumentar a sustentabilidade dos agroecossistemas, trazendo benefícios para as culturas de interesse econômico, o solo e o ambiente, mostrando-se uma alternativa economicamente viável e ecologicamente sustentável. Para adubação verde, as leguminosas são as preferidas, pelo fato de as raízes dessas plantas fixarem $\mathrm{N}_{2}$ atmosférico, em associação com bactérias diazotrófícas, enriquecendo o solo com esse nutriente. Além do N, as leguminosas produzem biomassa geralmente rica em $\mathrm{P}, \mathrm{Ke} \mathrm{Ca}$ e apresentam sistema radicular bem ramificado e profundo, que permite a reciclagem dos nutrientes no solo que serão assimilados pela planta, que, ao se decompor, irá torná-los disponíveis para as culturas econômicas (Costa, 1993).

Assim, no manejo de plantas de cobertura, a compreensão dos fatores que regulam a decomposição pode assumir importante papel no manejo das culturas, possibilitando a elaboração de técnicas de cultivo que melhorem a utilização de nutrientes contidos nos resíduos vegetais que formam a serapilheira. A decomposição é regulada pela interação de três grupos de variáveis: as condições físicoquímicas do ambiente, as quais são controladas pelo clima e pelas características edáficas do sítio; a qualidade (orgânica e nutricional) do substrato, que determina sua degradabilidade; e a natureza da comunidade decompositora, os macro e microrganismos (Heal et al., 1997; Correia \& Andrade, 1999). De modo geral, o clima controla o processo de decomposição em escala regional, enquanto a composição química domina o processo em escala local (Berg, 2000).

Diversos trabalhos têm sido desenvolvidos para avaliar o potencial de decomposição e mineralização de várias espécies de plantas de cobertura, especialmente em plantio direto (Bertol et al., 1998; Torres et al., 2005) e pastagens consorciadas (Oliveira et al., 2003). Entretanto, na fruticultura, particularmente na região noroeste fluminense, esses estudos são escassos. 
Em razão disso, o objetivo deste trabalho foi estimar as taxas de decomposição e liberação de nutrientes de resíduos culturais provenientes de plantas de coberturas na cultura do maracujá.

\section{MATERIAL E MÉTODOS}

O trabalho foi realizado na Fazenda Rio das Flores, Miracema, noroeste do Estado do Rio de Janeiro (21 ${ }^{\circ} 21^{\prime} \mathrm{S} \mathrm{e} 42^{\circ} 51^{\prime} \mathrm{W}$ ), no período de outubro de 2001 a agosto de 2002, em Argissolo Vermelho-Amarelo eutrófico franco-argilo-arenoso, com declividade de $30 \mathrm{~cm} \mathrm{~m}^{-1}$ (Paulucio, 2003). A precipitação e temperatura média anual foram de $1.400 \mathrm{~mm}$ e $25^{\circ} \mathrm{C}$, respectivamente.

Mediram-se a decomposição e a liberação de C, N, $\mathrm{P}, \mathrm{K}, \mathrm{Ca}$ e $\mathrm{Mg}$ de feijão-de-porco (Canavalia ensiformis (L.) DC.), de amendoim forrageiro acesso CIAT 1734 (Arachis pintoi Krap. \& Greg.), de siratro (Macroptilium atropurpureum (DC.) Urb.), de cudzu tropical (Pueraria phaseoloides (Roxb) Benth) e de vegetação espontânea (a espécie dominante observada na área foi Brachiaria brizantha (Hochst. ex A. Rich.) Stapf.) em dois tratamentos (com e sem adubação). O delineamento experimental adotado foi o de blocos ao acaso, com quatro repetições, num total de 24 parcelas de $35,1 \mathrm{~m}^{2}(13,0 \times 2,7 \mathrm{~m})$

No plantio, o tratamento de Brachiaria brizantha adubada e as leguminosas receberam $44 \mathrm{~kg} \mathrm{ha}^{-1} \mathrm{de}$ superfosfato simples, $33 \mathrm{~kg} \mathrm{ha}^{-1}$ de cloreto de potássio e $40 \mathrm{~kg} \mathrm{ha}^{-1}$ de FTE-BR 12 (Perin et al., 1998). O plantio das leguminosas foi feito em outubro de 2001, entre fileiras da cultura do maracujazeiro de 2,5 anos de idade. A densidade de plantio foi de 30 sementes por metro linear para siratro, cudzu e amendoim forrageiro (Guerra \& Teixeira, 1997) e de oito sementes por metro linear para feijão-de-porco (Calegari et al., 1993).

O corte dos adubos verdes foi feito quando o feijãode-porco atingiu $70 \%$ de floração e quando o amendoim forrageiro, siratro e cudzu atingiram $90 \%$ de cobertura do solo, aproximadamente 145 dias após plantio. O corte dos tratamentos de braquiária (com e sem adubação) foi na mesma época do das leguminosas.

Uma porção de $30 \mathrm{~g}$ de cada planta de cobertura recém-cortada foi coletada e colocada em sacos de decomposição de $2 \mathrm{~mm}$ de malha (náilon), com dimensões de 20 x $20 \mathrm{~cm}$ (Anderson \& Ingram, 1996). A massa seca referente ao tempo inicial (Ti) foi obtida mediante um fator de correção após secagem de amostras em estufa a $60^{\circ} \mathrm{C}$ até peso constante. Em cada parcela experimental, para cada planta de cobertura prepararam-se 28 sacos de decomposição, que foram colocados sobre a superfície do solo, em março de 2002. No período de 140 dias, foram coletados quatro sacos, nos intervalos de tempo de $0,7,15,30$, 60,90 e 140 dias, tendo sido as amostras limpas, para retirar o solo aderido, secas e pesadas.
Nas amostras, determinaram-se os teores de $\mathrm{P}$ (colorimetricamente, pelo método do complexo fosfomolíbdico, reduzido com ácido ascórbico, modificado por Braga \& Defelipo, 1974), de K (fotometria de chama), de Ca e Mg (espectrofotometria de absorção atômica em chama), após digestão nítricoperclórica, e de N, pelo método Kjeldahl, descritos por Bataglia et al. (1983). O teor de C foi obtido por oxidação com $\mathrm{K}_{2} \mathrm{Cr}_{2} \mathrm{O}_{7} 1,25 \mathrm{~mol} \mathrm{~L}^{-1} \mathrm{em}$ meio ácido, com uso de sacarose como padrão; e para polifenóis solúveis totais (polifenóis solúveis, taninos hidrolisáveis e condensados, e polifenóis não-tanínicos) empregou-se o método Folin-Denis em meio básico, a partir de uma amostra de $100 \mathrm{mg}$ misturada a $40 \mathrm{~mL}$ de metanol $50 \%$, mantidos à temperatura de $80^{\circ} \mathrm{C}$ durante uma hora, usando como padrão o ácido tânico, descrito por Anderson \& Ingram (1996).

A matéria seca decomposta em função do tempo foi calculada pela diferença entre o peso original $(30 \mathrm{~g})$ e o peso determinado ao final de cada período de decomposição, obtendo-se, assim, o percentual de peso seco remanescente. Com esses dados, estimaram-se as taxas da decomposição e de liberação diária de nutrientes $(k)$ por diversos modelos de regressão comumente usados em estudos de decomposição (Wieder \& Lang, 1982). O modelo que proporcionou melhor ajuste para todas as espécies foi o exponencial de primeira ordem, $\mathrm{Mt}=\mathrm{Mi} \mathrm{e}^{-k \mathrm{~T}}$ (Olson, 1963), em que Mt é o percentual de peso seco remanescente após t anos e Mi é $100 \%$ quando t é igual a zero. Obtevese, assim, para cada planta de cobertura, em cada parcela experimental, uma equação. Com base no modelo ajustado, calculou-se o tempo de meia-vida $\left(\mathrm{t}_{1 / 2}=0,693 / \mathrm{k}\right)$. O $\mathrm{t}_{1 / 2}$ é o tempo necessário para que ocorra $50 \%$ de decomposição e de liberação de nutrientes. Na análise de variância das taxas de decomposição e de liberação de nutrientes, aplicou-se o teste F para significância. Para comparar as médias, foi usado o teste de Tukey a $5 \%$.

\section{RESULTADOS E DISCUSSÃO}

O feijão-de-porco e o amendoim forrageiro apresentaram as maiores taxas de decomposição de matéria seca, diferindo significativamente das demais coberturas vegetais (Quadro 1). Aproximadamente $50 \%$ de perda da matéria seca dessas duas leguminosas ocorreu num período de 60 dias. As leguminosas siratro e cudzu não diferiram significativamente dos tratamentos de braquiária adubada e não-adubada. Essas coberturas apresentaram tempo de meia-vida superior a 100 dias. A adubação não influenciou significativamente a taxa de decomposição da braquiária. Os valores das taxas de liberação de C, nutrientes e polifenóis solúveis variaram significativamente entre as coberturas vegetais (Quadro 1). O feijão-de-porco e o amendoim forrageiro apresentaram as maiores taxas de liberação 
de $\mathrm{C}$, diferindo das outras espécies. As taxas de liberação de N, P, Ca e Mg também foram maiores no feijão-de-porco. Contudo, apenas para $\mathrm{N}$ esta espécie diferiu significativamente das demais coberturas. Somente para $\mathrm{P} \mathrm{e} \mathrm{Mg} \mathrm{o} \mathrm{feijão-de-porco} \mathrm{e} \mathrm{o} \mathrm{amendoim}$ forrageiro foram estatisticamente similares entre si. Para Ca, o feijão-de-porco só diferiu significativamente do siratro. Entretanto, o feijão-de-porco apresentou uma das menores taxas de liberação de $\mathrm{K}$, diferindo significativamente apenas do amendoim forrageiro, que mostrou a maior taxa, porém igual ao siratro e braquiária com e sem adubação.

Os tratamentos de braquiária tenderam a apresentar as menores taxas de liberação para todos os nutrientes, à exceção de $\mathrm{K}$. Apenas para $\mathrm{P}$ houve efeito significativo e positivo da adubação. De maneira geral, para quase todos os nutrientes, o siratro e o cudzu tenderam a não diferir significativamente dos tratamentos de braquiária, mostrando valores de taxas de liberação intermediários entre a gramínea e o feijãode-porco e o amendoim forrageiro (Quadro 1). Para polifenóis solúveis, destacou-se o amendoim forrageiro, que mostrou taxa de liberação cerca de 10 vezes superior à das demais espécies; entretanto, essa leguminosa só diferiu significativamente do cudzu, provavelmente devido ao elevado coeficiente de variação dessa fração orgânica na análise de variância (Quadro 1).

Para todas as coberturas vegetais, os maiores valores médios de taxa de liberação foram de $\mathrm{K}$ e polifenóis. A rápida liberação de $\mathrm{K}$ ocorrida nos primeiros 30 dias de decomposição indica que a lixiviação seria um dos principais mecanismos de transferência de K para o solo, uma vez que ele não é componente estrutural de qualquer composto das plantas e a mineralização não é um pré-requisito para sua liberação (Gama-Rodrigues \& Barros, 2002; Costa et al., 2005). Por outro lado, certa quantidade de $\mathrm{Ne}$ $P$ rapidamente liberados no estádio inicial de decomposição dos resíduos culturais estaria associada à perda de frações desses nutrientes solúveis em água (Giacomini et al., 2003; Aita \& Giacomini, 2003). Procedimento análogo também foi verificado para o C, Ca e Mg (Gama-Rodrigues et al., 1999).

Assim como o K, a rápida liberação de polifenóis solúveis nos estádios iniciais de decomposição dos resíduos vegetais estaria também associada ao mecanismo de lixiviação (Quadro 1). A lixiviação tem sido considerada o principal mecanismo de transferência de polifenóis solúveis para o solo em diversos resíduos agrícolas e florestais (Handayanto et al., 1997; Hättenschwiler \& Vitousek, 2000; Costa et al., 2005).

A seqüência, em ordem decrescente, da taxa de liberação média dos resíduos vegetais avaliados foi de polifenóis $>\mathrm{K}>\mathrm{N}>\mathrm{Mg}>\mathrm{P}>\mathrm{Ca}$ (Quadro 1).

No quadro 2 são mostradas as correlações das taxas de liberação entre as variáveis analisadas. As liberações de $\mathrm{C}, \mathrm{N}, \mathrm{P}, \mathrm{Ca}$ e $\mathrm{Mg}$ estão associadas positivamente à taxa de decomposição da matéria seca. $\mathrm{O} \mathrm{C}$ também se correlacionou positivamente com $\mathrm{N}$, $\mathrm{P}, \mathrm{Ca}$ e Mg. Isso indica que a liberação desses nutrientes acompanharia a perda de massa e, ou, a mineralização de C. Em povoamentos de eucalipto a liberação de N e Mg foi associada à perda de massa (Gama-Rodrigues $\&$ Barros, 2002; Costa et al., 2005).

Quadro 1. Valores estimados da taxa de decomposição e de liberação de nutrientes e de polifenóis (K) e tempo de meia-vida $\left(T_{1 / 2}\right)$ de resíduos vegetais

\begin{tabular}{|c|c|c|c|c|c|c|c|c|}
\hline Tratamento & Matéria seca ${ }^{(1)}$ & $\mathbf{C}$ & $\mathbf{N}$ & $\mathbf{P}$ & $\mathbf{K}$ & $\mathbf{C a}$ & Mg & Polifenóis \\
\hline Feijão-de-porco & $0,0133 \mathrm{a}$ & $0,0150 \mathrm{a}$ & 0,0162 a & $0,0104 \mathrm{a}$ & $0,0283 \mathrm{~b}$ & 0,0122 a & $0,0140 \mathrm{a}$ & $0,0465 \mathrm{ab}$ \\
\hline Amendoim forrageiro & 0,0116 a & $0,0115 \mathrm{ab}$ & $0,0108 \mathrm{~b}$ & $0,0101 \mathrm{a}$ & $0,0670 \mathrm{a}$ & $0,0083 \mathrm{ab}$ & $0,0130 \mathrm{a}$ & $0,3557 \mathrm{a}$ \\
\hline Siratro & $0,0066 \mathrm{~b}$ & $0,0071 \mathrm{~b}$ & $0,0088 \mathrm{bc}$ & $0,0068 \mathrm{~b}$ & $0,0452 \mathrm{ab}$ & $0,0025 \mathrm{~b}$ & $0,0071 \mathrm{~b}$ & $0,0358 \mathrm{ab}$ \\
\hline Cudzu & $0,0053 \mathrm{~b}$ & $0,0072 \mathrm{~b}$ & $0,0086 \mathrm{bc}$ & $0,0058 \mathrm{~b}$ & $0,0250 \mathrm{~b}$ & $0,0052 \mathrm{ab}$ & $0,0067 \mathrm{~b}$ & $0,0255 \mathrm{~b}$ \\
\hline Braquiária adubada & $0,0064 \mathrm{~b}$ & $0,0065 \mathrm{~b}$ & $0,0053 \mathrm{c}$ & $0,0062 \mathrm{~b}$ & $0,0325 \mathrm{ab}$ & $0,0035 \mathrm{ab}$ & $0,0057 \mathrm{~b}$ & $0,0590 \mathrm{ab}$ \\
\hline \multirow[t]{2}{*}{ Braquiária não-adubada } & $0,0060 \mathrm{~b}$ & $0,0050 \mathrm{~b}$ & $0,0045 \mathrm{c}$ & $0,0026 \mathrm{c}$ & $0,0496 \mathrm{ab}$ & $0,0051 \mathrm{ab}$ & $0,0047 \mathrm{~b}$ & $0,0832 \mathrm{ab}$ \\
\hline & & & - & $-\mathrm{T}_{1 / 2}(\mathrm{dia}$ & -1) & & & \\
\hline Feijão-de-porco & 52 & 46 & 43 & 67 & 24 & 57 & 49 & 15 \\
\hline Amendoim forrageiro & 60 & 60 & 64 & 69 & 10 & 83 & 53 & 2 \\
\hline Siratro & 105 & 98 & 79 & 102 & 15 & 277 & 98 & 19 \\
\hline Cudzu & 131 & 96 & 81 & 119 & 28 & 133 & 103 & 27 \\
\hline Braquiária adubada & 108 & 107 & 131 & 112 & 21 & 198 & 122 & 12 \\
\hline Braquiária não-adubada & 115 & 139 & 154 & 267 & 14 & 136 & 147 & 8 \\
\hline
\end{tabular}

(1) Médias seguidas de letras iguais, na mesma coluna, não diferem entre si pelo teste de Tukey a $5 \%$. 
A liberação de $\mathrm{N}$ se correlacionou com a de $\mathrm{P}, \mathrm{Ca}$ e Mg. Já a liberação de Mg se correlacionou com a de $\mathrm{P}$ e Ca. Essas correlações seriam devido ao fato de esses nutrientes, em sua maior parte, serem componentes estruturais do tecido vegetal. Entretanto, a liberação de $\mathrm{K}$ se correlacionou positivamente apenas com a dos polifenóis (Quadro 2). A estreita correlação entre K e polifenóis pode ser atribuída à lixiviação como principal mecanismo de liberação deste elemento e da fração orgânica durante o processo de decomposição.

A qualidade nutricional e orgânica dos resíduos vegetais avaliados variou significativamente (Quadro 3). De maneira geral, o feijão-de-porco mostrou resíduo de melhor qualidade em razão dos maiores teores de N, P e Ca e menores valores das relações $\mathrm{C} / \mathrm{N}, \mathrm{C} / \mathrm{P}$ e polifenol/N. Para $\mathrm{K}$ não houve diferença significativa entre as espécies. A adubação não alterou significativamente a qualidade da braquiária.
As taxas de decomposição de matéria seca e de liberação de $\mathrm{C}$, de nutrientes e de polifenóis variaram em função da qualidade nutricional e orgânica do substrato referente ao início do estudo (Quadro 4). Os teores de $\mathrm{K}$ (negativamente), Ca e polifenóis solúveis (positivamente) mostraram relação linear significativa com a taxa de decomposição. Essas três variáveis também mostraram capacidade preditiva das taxas de liberação de C, P, Ca e Mg. Todavia, somente os teores de $\mathrm{K}$ e de Ca mostraram capacidade preditiva da taxa de liberação de $\mathrm{N}$. Os teores de $\mathrm{N}$ e $\mathrm{Mg}$ mostraram relação linear significativa com as taxas de liberação de $\mathrm{C}$ e $\mathrm{P}$, respectivamente. Apenas os teores de N, Ca e polifenóis apresentaram relação linear com as suas respectivas taxas de liberação. $\mathrm{O}$ teor de $\mathrm{C}$ mostrou relação linear negativa apenas com a taxa de liberação de polifenóis. Essa relação indica que substratos com alto teor de $\mathrm{C}$ teriam baixas concentrações de polifenóis solúveis e, por conseguinte,

Quadro 2. Correlações de Pearson entre as taxas de decomposição e de mineralização de resíduos vegetais

\begin{tabular}{|c|c|c|c|c|c|c|c|c|}
\hline & $\mathbf{M S} \mathbf{S}^{(1)}$ & C & $\mathbf{N}$ & $\mathbf{P}$ & $\mathbf{K}$ & $\mathrm{Ca}$ & Mg & $\mathrm{POL}^{(2)}$ \\
\hline MS & 1 & $0,958^{* *}$ & $0,857^{*}$ & $0,871^{*}$ & 0,224 & $0,897^{* *}$ & $0,966^{* * *}$ & 0,483 \\
\hline $\mathrm{C}$ & & 1 & $0,957 * *$ & $0,914 * *$ & $-0,010$ & $0,911 * *$ & $0,974^{* * *}$ & 0,313 \\
\hline $\mathrm{N}$ & & & 1 & $0,865^{*}$ & $-0,139$ & $0,838^{*}$ & $0,913^{* *}$ & 0,129 \\
\hline $\mathrm{P}$ & & & & 1 & 0,107 & 0,688 & $0,937^{* *}$ & 0,444 \\
\hline K & & & & & 1 & $-0,027$ & 0,195 & $0,844^{*}$ \\
\hline $\mathrm{Ca}$ & & & & & & 1 & $0,869^{*}$ & 0,292 \\
\hline $\mathrm{Mg}$ & & & & & & & 1 & 0,504 \\
\hline
\end{tabular}

${ }^{(1)}$ MS: matéria seca. ${ }^{(2)}$ POL: polifenóis. *, ** e ${ }^{* * *}$ : Significativos a 0,1, 1 e $5 \%$, respectivamente.

Quadro 3. Características químicas iniciais de resíduos vegetais

\begin{tabular}{|c|c|c|c|c|c|c|}
\hline Variável & $\begin{array}{l}\text { Feijão-de- } \\
\text { porco }^{(1)}\end{array}$ & $\begin{array}{l}\text { Amendoim } \\
\text { forrageiro }\end{array}$ & Siratro & Cudzu & $\begin{array}{c}\text { Braquiária } \\
\text { adubada }\end{array}$ & $\begin{array}{c}\text { Braquiária } \\
\text { não-adubada }\end{array}$ \\
\hline $\mathrm{C}\left(\mathrm{g} \mathrm{kg}^{-1}\right)^{(2)}$ & $352 \quad a b$ & 314 & 363 & 349 & 343 & $353 \mathrm{ab}$ \\
\hline $\mathrm{N}\left(\mathrm{g} \mathrm{kg}^{-1}\right)$ & 38,6 a & 20,9 & $27,2 \quad b$ & 25,8 & $11,0 \quad d$ & 10,7 \\
\hline $\mathrm{P}\left(\mathrm{g} \mathrm{kg}^{-1}\right)$ & $1,68 \mathrm{a}$ & $0,92 \mathrm{~b}$ & $1,87 \mathrm{a}$ & $1,43 \mathrm{ab}$ & $1,04 \mathrm{~b}$ & $0,96 \mathrm{~b}$ \\
\hline $\mathrm{K}\left(\mathrm{g} \mathrm{kg}^{-1}\right)$ & 17,5 a & $16,0 \mathrm{a}$ & $22,2 \quad \mathrm{a}$ & 19,8 a & 21,3 a & 22,5 a \\
\hline $\mathrm{Ca}\left(\mathrm{g} \mathrm{kg}^{-1}\right)$ & $20,0 \quad$ a & $13,8 \quad b$ & 8,3 & 10,5 & 2,8 & $2,7 \quad \mathrm{~d}$ \\
\hline $\mathrm{Mg}\left(\mathrm{g} \mathrm{kg}^{-1}\right)$ & $2,90 \mathrm{abc}$ & 3,72 a & $3,50 \mathrm{ab}$ & 2,50 & $2,30 \quad \mathrm{c}$ & $1,90 \quad \mathrm{c}$ \\
\hline POL $\left(\mathrm{g} \mathrm{kg}^{-1}\right)^{(3)}$ & 20,0 & 23,1 & $13,7 \quad b$ & 14,5 & 13,2 & 13,5 \\
\hline $\mathrm{C} / \mathrm{N}$ & 9 & 15 & 13 & 14 & 31 & 33 \\
\hline $\mathrm{C} / \mathrm{P}$ & 210 & 341 & 194 & 244 & 329 & 368 \\
\hline $\mathrm{POL} / \mathrm{N}$ & 0,52 & $1,10 \mathrm{a}$ & 0,50 & 0,56 & $1,20 \mathrm{a}$ & $1,27 \mathrm{a}$ \\
\hline
\end{tabular}

${ }^{(1)}$ Médias seguidas de letras iguais, na mesma linha, não diferem entre si pelo teste de Tukey a $5 \%$. ${ }^{(2)}$ Teores totais. ${ }^{(3)}$ Polifenóis. 
Quadro 4. Regressões lineares das taxas de decomposição e de liberação de carbono, fósforo, potássio, cálcio, magnésio e polifenóis de resíduos vegetais de acordo com seus atributos químicos

\begin{tabular}{|c|c|}
\hline $\mathrm{MS}^{(1)}=0,0291-0,001052^{*}(\mathrm{TK})$ & $\mathrm{R}^{2}=0,694$ \\
\hline $\mathrm{MS}=0,0041+0,000423^{*}(\mathrm{TCa})$ & $\mathrm{R}^{2}=0,707$ \\
\hline $\mathrm{MS}=-0,0035+0,00072 * *(\mathrm{TPOL})$ & $\mathrm{R}^{2}=0,808$ \\
\hline $\mathrm{C}=0,0026+0,000272^{*}(\mathrm{TN})$ & $\mathrm{R}^{2}=0,588$ \\
\hline $\mathrm{C}=0,3254-0,001198^{*}(\mathrm{TK})$ & $\mathrm{R}^{2}=0,719$ \\
\hline $\mathrm{C}=0,0035+0,000532^{* *}(\mathrm{TCa})$ & $\mathrm{R}^{2}=0,892$ \\
\hline $\mathrm{C}=-0,0036+0,000756^{*}(\mathrm{TPOL})$ & $\mathrm{R}^{2}=0,712$ \\
\hline $\mathrm{N}=0,0008+0,000365 * *(\mathrm{TN})$ & $\mathrm{R}^{2}=0,845$ \\
\hline $\mathrm{N}=0,0324-0,001179^{*}(\mathrm{TK})$ & $\mathrm{R}^{2}=0,554$ \\
\hline $\mathrm{N}=0,003+0,000622^{* *}(\mathrm{TCa})$ & $\mathrm{R}^{2}=0,969$ \\
\hline $\mathrm{N}=0,0158-0,000353^{*}(\mathrm{C} / \mathrm{N})$ & $\mathrm{R}^{2}=0,724$ \\
\hline $\mathrm{P}=0,0256-0,000941^{*}(\mathrm{TK})$ & $\mathrm{R}^{2}=0,737$ \\
\hline $\mathrm{P}=0,0034+0,000374^{*}(\mathrm{TCa})$ & $\mathrm{R}^{2}=0,734$ \\
\hline $\mathrm{P}=-0,0017+0,003113^{*}(\mathrm{TMg})$ & $\mathrm{R}^{2}=0,567$ \\
\hline $\mathrm{P}=-0,0025+0,000583^{*}(\mathrm{TPOL})$ & $\mathrm{R}^{2}=0,702$ \\
\hline $\mathrm{P}=0,0111-0,000211^{*}(\mathrm{C} / \mathrm{N})$ & $\mathrm{R}^{2}=0,546$ \\
\hline $\mathrm{Ca}=0,0273-0,001066^{*}(\mathrm{TK})$ & $\mathrm{R}^{2}=0,635$ \\
\hline $\mathrm{Ca}=0,0017+0,000454^{*}(\mathrm{TCa})$ & $\mathrm{R}^{2}=0,724$ \\
\hline $\mathrm{Ca}=-0,0049+0,000677^{*}(\mathrm{TPOL})$ & $\mathrm{R}^{2}=0,635$ \\
\hline $\mathrm{Mg}=0,0354-0,001349 * *(\mathrm{TK})$ & $\mathrm{R}^{2}=0,829$ \\
\hline $\mathrm{Mg}=0,0032+0,000546^{* *}(\mathrm{TCa})$ & $\mathrm{R}^{2}=0,854$ \\
\hline $\mathrm{Mg}=-0,0058+0,000879 * *(\mathrm{TPOL})$ & $\mathrm{R}^{2}=0,874$ \\
\hline $\mathrm{POL}^{(2)}=2,5164-0,006995^{* *}(\mathrm{TC})$ & $\mathrm{R}^{2}=0,849$ \\
\hline $\mathrm{POL}=-0,2746+0,022979^{*}(\mathrm{TPOL})$ & $\mathrm{R}^{2}=0,583$ \\
\hline
\end{tabular}

${ }^{(1)}$ MS: matéria seca. ${ }^{(2)}$ PDL: polifenóis.

menores taxas de liberação dessas frações orgânicas por lixiviação durante o processo de decomposição.

A relação negativa do teor de $\mathrm{K}$ com as taxas de decomposição de matéria seca e de liberação de $C, N$, $\mathrm{P}, \mathrm{Ca}$ e Mg não indica aparentemente uma relação de causa-efeito, mas sim que substratos com altos teores de $\mathrm{K}$ possuiriam maior quantidade de formas de $\mathrm{C}$ mais recalcitrantes e, portanto, com menores taxas de decomposição e mineralização. Desse modo, o teor de $K$ poderia ser um indicador indireto do grau de recalcitrância de substratos submetidos à decomposição, pois a liberação deste elemento dá-se predominantemente pelo mecanismo de lixiviação.

Por sua vez, a relação positiva do teor de polifenóis com as taxas de decomposição de matéria seca e de liberação de $\mathrm{C}$ indicaria que parte significativa da perda de massa ou liberação de $\mathrm{C}$ seria devida à lixiviação e que a redução de polifenóis resultaria, por conseguinte, numa diminuição da concentração de compostos orgânicos recalcitrantes à ação dos organismos decompositores, o que acarretaria, por sua vez, alta taxa de decomposição ou liberação de $\mathrm{C}$ do material remanescente. Raciocínio análogo poderia ser aplicado às relações positivas entre as taxas de liberação de $\mathrm{P}, \mathrm{Ca}$ e $\mathrm{Mg}$ e o teor de polifenóis (Quadro 4). Contudo, essa fração orgânica não mostrou relação significativa com a taxa de liberação de $\mathrm{N}$, apesar de positiva.

A lixiviação de polifenóis solúveis no estádio inicial da decomposição resultaria em alta taxa de $\mathrm{N}$ mineralizado pela redução da quantidade desse nutriente complexado por essa fração orgânica (Handayanto et al., 1997).

A relação C/N mostrou capacidade preditiva apenas para as taxas de liberação de N e P (Quadro 4). Quanto maior a relação $\mathrm{C} / \mathrm{N}$, menor seria a liberação desses nutrientes por ação dos microrganismos decompositores. Desse modo, a relação $\mathrm{C} / \mathrm{N}$ expressaria o grau de recalcitrância do substrato no processo de decomposição. Contudo, nenhuma relação significativa foi obtida entre as relações $\mathrm{C} / \mathrm{P}$ e polifenol/ $\mathrm{N}$ e as taxas de decomposição de matéria seca e liberação dos elementos minerais.

Diversos índices de qualidade têm sido propostos como bons preditores da taxa de decomposição e liberação de nutrientes, principalmente de $\mathrm{N}$ e $\mathrm{P}$, a partir da determinação de alguns constituintes orgânicos e nutricionais. Desses, os reconhecidos como de maior influência são as relações $\mathrm{C} / \mathrm{N}, \mathrm{C} / \mathrm{P}$, polifenol/ $\mathrm{N}$, lignina/N, lignina/P, (lignina + polifenol) $/ \mathrm{N}$ e (lignina + celulose)/N (Palm \& Sanchez, 1991; Handayanto et al., 1997; Palm et al., 2001; Giacomini et al., 2003; Oliveira et al., 2003; Gama-Rodrigues et al., 2003; Albrecht et al., 2004). Todos esses índices são válidos, porém cada um apresenta vantagens e limitações de uso (Cadisch \& Giller, 1997). Os modelos preditivos obtidos são, de maneira geral, de aplicabilidade específica de cada local (ou sítio), pois são modelos empíricos, ou seja, restritos à condição edafoclimática, ao tipo de sistema de produção, às espécies associadas e ao manejo (Gama-Rodrigues, 2004). Apesar disso, é possível obter razoável precisão de predição das taxas de decomposição dos materiais vegetais que são normalmente usados em sistemas como adubo verde. Em geral, valores elevados para todos esses índices propostos acarretam baixa decomposição e liberação de $\mathrm{N}$ e P.

Entretanto, no presente trabalho, o fato de as taxas de decomposição de matéria seca e de liberação de $\mathrm{C}$, $\mathrm{N}, \mathrm{P}$ e Mg terem variado principalmente em função dos teores de $\mathrm{K}$ e $\mathrm{Ca}$ (Quadro 4) revela que esses nutrientes, e não o $\mathrm{N} \mathrm{e} \mathrm{P}$, seriam os fatores reguladores mais limitantes para decomposição e mineralização dos resíduos das espécies avaliadas, nas condições estudadas. Evidências do papel relevante do $\mathrm{Ke} \mathrm{Ca}$ 
no processo de decomposição de resíduos agrícolas e florestais têm sido relatadas (Gama-Rodrigues et al., 1999; Giacomini et al., 2003).

Assim, as distintas taxas de decomposição e liberação de nutrientes das espécies avaliadas no presente trabalho mostraram a necessidade de estudos futuros do manejo de resíduos vegetais como fonte de nutrientes na cultura do maracujá. Desse modo, devido à elevada taxa de liberação de $\mathrm{K}$ dos resíduos culturais, parte dele poderá ser perdida no solo pela lixiviação, por ter sido liberado antes do período de maior demanda pela planta, que ocorre na fase de floração. Hipótese similar, mas de sentido inverso, valeria para a baixa liberação de $\mathrm{Ca}$, no qual a disponibilização do elemento ocorreria após a fase de maior demanda nutricional do maracujá.

Nesse sentido, a decomposição da biomassa das plantas de cobertura pode ser manejada para melhorar a absorção e utilização de nutrientes fornecidos à cultura do maracujá. Para isso, podem ser estabelecidas duas estratégias: regular as taxas de liberação dos nutrientes para melhorar a sincronização do suprimento do nutriente com a demanda da planta; e proporcionar ambiente mais favorável ao crescimento da planta (Mafongoya et al., 1997). A primeira estratégia é de natureza imediata (curto período de tempo), enquanto a segunda envolve melhoria de longo tempo, freqüentemente associada ao aumento e manutenção da matéria orgânica do solo.

Regular as taxas de liberação de nutrientes pela mistura de resíduos de plantas de cobertura de distinta qualidade química tem se mostrado uma técnica muito promissora, especialmente em sistemas de plantio direto (Aita \& Giacomini, 2003), de pastagens consorciadas (Oliveira et al., 2003), de florestas mistas (Gama-Rodrigues et al., 2003) e de agroflorestas (GamaRodrigues, 2004). Na mistura de resíduos, a decomposição do material mais recalcitrante aumenta, mas diminui o de alta qualidade (Gama-Rodrigues et al., 2003). Dessa forma, isso possibilitaria que a liberação de nutrientes fosse sincronizada com a fase de maior demanda nutricional da cultura associada. Assim, a melhora na sincronização aumentaria a eficiência de uso de nutrientes, minimizando suas perdas e também a racionalização da aplicação de fertilizantes minerais - quantidade, localização e época (Myers et al., 1997). A sincronização, portanto, pode ser efetuada (1) pela manipulação da demanda nutricional da cultura mediante a época de plantio e seleção da cultura (espécie ou variedade) e (2) pela manipulação da liberação de nutrientes por meio do manejo da biomassa, como descrito anteriormente.

\section{CONCLUSÕES}

1. As coberturas vegetais apresentaram distintas taxas de decomposição e de liberação de nutrientes, sendo o feijão-de-porco a espécie que mostrou resíduo de melhor qualidade química.

2. $\mathrm{O} \mathrm{K}$ e $\mathrm{Ca}$ foram os fatores reguladores mais limitantes das taxas de decomposição e de liberação de nutrientes dos resíduos vegetais.

\section{AGRADECIMENTO}

Ao Sr. Carlos Roberto de Medeiros, pelo apoio logístico de campo na realização deste trabalho.

\section{LITERATURA CITADA}

AITA, C. \& GIACOMINI, S.J. Decomposição e liberação de nitrogênio de resíduos culturais de plantas de cobertura de solo solteiras e consorciadas. R. Bras. Ci. Solo, 27:601612,2003

ALBRECHT, A.; CADISCH, G.; BLANCHART, E.; SITOMPUL, S.M. \& VANLAUWE, B. Below-ground inputs: relationships with soil quality, soil $\mathrm{C}$ storage and soil structure. In: van NOORDWIJK, M.; CADISCH, G. \& ONG, C.K., orgs. Below-ground interactions in tropical agroecosystems: Concepts and models with multiple plant components. Wallingford, CAB International, 2004. p.193207.

ANDERSON, J.N. \& INGRAM, J.S.I. Tropical soil biology and fertility: A handbook of methods. Wallingford, $\mathrm{CAB}$ International, 1996. $171 \mathrm{p}$.

BATAGLIA, O.C.; FURLANI, A.M.C.; TEIXEIRA, J.P.F.; FURLANI, P.R. \& GALLO, J.R. Métodos de análise química de plantas. Campinas, Instituto Agronômico de Campinas, 1983. 48p. (Boletim Técnico, 78)

BERG, B. Litter decomposition and organic matter turnover in northern forest soil. For. Ecol. Manag., 133:13-22, 2000.

BERTOL, I.; CIPRANDI, O.; KURTZ, C. \& BAPTISTA, A.S. Persistência de resíduos culturais de aveia e milho sobre a superfície do solo em semeadura direta. R. Bras. Ci. Solo, 22:705-712, 1998.

BRAGA, J.M. \& DEFELIPO, B.V. Determinação espectrofotométrica de fósforo em extrato de solo e material vegetal. R. Ceres, 21:73-85, 1974.

CADISCH, G. \& GILLER, K.E. Driven by nature: Plant litter quality and decomposition. Wallingford, $\mathrm{CAB}$ International, 1997. 409p.

CALEGARI, A.; MONDARDO, A.; BULIZANI, E.A.; COSTA, M.B.B.; MIYSAKA, S. \& AMADO,T.J.C. Aspectos gerais da adubação verde. In: COSTA, M.B.B., org. Adubação verde no sul do Brasil. Rio de Janeiro, AS-PTA, 1993. p.155

CORREIA, M.E.F. \& ANDRADE, A.G. Formação de serapilheira e ciclagem de nutrientes. In: SANTOS, G.A. \& CAMARGO, F.A.O., orgs. Fundamentos da matéria orgânica do solo: Ecossistemas tropicais e subtropicais. Porto Alegre, Gênesis, 1999. p.197-225. 
COSTA, G.S.; GAMA-RODRIGUES, A.C. \& CUNHA, G.M. Decomposição e liberação de nutrientes da serapilheira foliar em povoamentos de Eucalyptus grandis no norte fluminense. R. Árvore, 29:563-570, 2005.

COSTA, M.B.B. Adubação verde no sul do Brasil. Rio de Janeiro, AS-PTA, 1993. 346p.

GAMA-RODRIGUES, A.C.; BARROS, N.F \& SANTOS, M.R Decomposição e liberação de nutrientes do folhedo de espécies florestais nativas em plantios puros e mistos no sudeste da Bahia, Brasil. R. Bras. Ci. Solo, 27:1021-1031, 2003.

GAMA-RODRIGUES, A.C. Ciclagem de nutrientes em sistemas agroflorestais na região tropical: Funcionalidade e sustentabilidade. In: MÜLLER, M.W.; GAMARODRIGUES, A.C.; BRANDÃO, I.C.S.F.L. \& SERÓDIO, M.H.C.F., orgs. Sistemas agroflorestais, tendência da agricultura ecológica nos trópicos: Sustento da vida e sustento de vida. Ilhéus, SBSAF/CEPLAC/UENF, 2004. p.64-84.

GAMA-RODRIGUES, A.C. \& BARROS, N.F. Ciclagem de nutrientes em floresta natural e em plantios de eucalipto e de dandá no sudeste da Bahia, Brasil. R. Árvore, 26:193207, 2002.

GAMA-RODRIGUES, A.C. \& MAY, P. Sistemas agroflorestais e o planejamento do uso da terra: Experiência na região norte fluminense, RJ. In: MACÊDO, J.L.V.; WANDELLI, E.V. \& SILVA JÚNIOR, J.P., orgs. Sistemas agroflorestais: Manejando a biodiversidade e compondo a paisagem rural. Manaus, Embrapa, 2001. p.130-136.

GAMA-RODRIGUES, A.C.; BARROS, N.F. \& MENDONÇA, E.S. Alterações edáficas sob plantios puros e misto de espécies florestais nativas do sudeste da Bahia, Brasil. $R$. Bras. Ci. Solo, 23:581-592, 1999.

GIACOMINI, S.J.; AITA, C.; HÜBNER, A.P.; LUNKES, A.; GUIDINI, E. \& AMARAL, E.B. Liberação de fósforo e potássio durante a decomposição de resíduos culturais em plantio direto. Pesq. Agropec. Bras., 38:1097-1104, 2003.

GUERRA, J.G.M. \& TEIXEIRA, M.G. Avaliação inicial de algumas leguminosas perenes para utilização como cobertura viva permanente de solo. Seropédica, Embrapa Agrobiologia, 1997. 6p. (Comunicado Técnico, 16)

HANDAYANTO, E.; CADISCH, G. \& GILLER, K.E. Regulating $\mathrm{N}$ mineralization from plant residues by manipulation of quality. In: CADISCH, G. \& GILLER, K.E., orgs. Driven by nature: Plant litter quality and decomposition. Wallingford, CAB International, 1997. p.175-185.

HÄTTENSCHWILER, S. \& VITOUSEK, P.M. The role of polyphenols in terrestrial ecosystem nutrient cycling. Tree, 15:238-243, 2000.

HEAL, O.W.; ANDERSON, J.M. \& SWIFT, M.J. Plant litter quality and decomposition: An historical overview. In CADISCH, G. \& GILLER, K.E., orgs. Driven by nature: Plant litter quality and decomposition. Wallingford, $\mathrm{CAB}$ International, 1997. p.3-30.
MAFONGOYA, P.L.; GILLER, K.E. \& PALM, C.A. Decomposition and nutrient release patterns of prunings and litter of agroforestry trees. Agrofor. Syst., 38:77-97, 1997.

MYERS, R.J.K.; van NOORDWIJK, M. \& VITYAKON, P. Synchrony of nutrient release and plant demand: Plant litter quality, soil environment and farmer management options. In: CADISCH, G. \& GILLER, K.E., orgs. Driven by nature: Plant litter quality and decomposition. Wallingford, CAB International, 1997. p.215-229.

OLIVEIRA, C.A.; MUZZI, M.R.S.; PURCINO, H.A.; MARIEL, I.E. \& SÁ, N.M.H. Decomposition of Archis pintoi and Hyparrhenia rufa litters in monoculture and intercropped systems under lowland soil. Pesq. Agropec. Bras., 38:1089$1195,2003$.

OLSON, J.S. Energy storage and the balance of producers and decomposers in ecological systems. Ecology, 44:322331,1963

PALM, C.A. \& SANCHEZ, P.A. Nitrogen release from the leaves of some tropical legumes as affected by their lignin and polyphenolic contents. Soil Biol. Biochem., 23:83-88, 1991.

PALM, C.A.; GACHENGO, C.N.; DELVE, R.J.; CADISCH, G. \& GILLER, K.E. Organic inputs for soil fertility management in tropical agroecosystems: Application of an organic resource database. Agric., Ecosyst. Environ., 83:27-42, 2001

PAULUCIO, V.O. Caracterização física e química do solo de uma toposseqüência sob diferentes coberturas vegetais no noroeste do Estado do Rio de Janeiro. Campos dos Goytacazes, Universidade Estadual do Norte Fluminense, 2003. 75p. (Tese de Mestrado).

PERIN, A.; TEIXEIRA, M.G. \& GUERRA, J.G.M. Avaliação inicial de algumas leguminosas herbáceas perenes para utilização como cobertura viva permanente de solo. II. Amendoim forrageiro, galáxia e centrosema. Seropédica, Embrapa Agrobiologia, 1998. 6p. (Comunicado Técnico, 28)

SIQUEIRA, J.O.; MOREIRA, F.M.S. \& LOPES, A.S. Interrelação fertilidade, biologia do solo e nutrição mineral de plantas: Base para um novo paradigma na agrotecnologia do século XXI. In: SIQUEIRA, J.O.; MOREIRA, F.M.S.; LOPES, A.S.; GUILHERME, L.R.G.; FAQUIN, V.; FURTINI NETO, A.E. \& CARVALHO, J.G., orgs. Interrelação fertilidade, biologia do solo e nutrição de plantas. Lavras, SBCS/UFLA/DCS, 1999. p.1-9.

TORRES, J.L.R.; PEREIRA, M.G.; ANDRIOLI, I.; POLIDORO, J.C. \& FABIAN, A.J. Decomposição e liberação de nitrogênio de resíduos culturais de plantas de cobertura em um solo de cerrado. R. Bras. Ci. Solo, 29:609-618, 2005 .

WIEDER, R.K. \& LANG, G.E. A critique of the analytical methods used in examinig decomposition data obtained from litter bags. Ecology, 63:1636-1642, 1982. 